\title{
Standards of care: Management of patients with nonvariceal upper gastrointestinal hemorrhage
}

\author{
Guido Van Rosendaal MD
}

\begin{abstract}
ARTICLE
Barkun A, Bardou M, Marshall JK, Nonvariceal Upper GI Bleeding Consensus Conference Group. Consensus recommendations for managing patients with nonvariceal upper gastrointestinal bleeding. Ann Intern Med 2003;139:843-57.
\end{abstract}

\section{SUMMARY}

This article is the outcome of a formal process of clinical guideline development involving 25 participants with expertise covering a wide range of disciplines and nationalities, as well as nonvoting observers representing Health Canada and seven industry sponsors. Twenty recommendations are presented, each specifying the degree of support and dissention expressed in the panel's votes. One hundred ninety-two references are cited. Early endoscopy and risk stratification are central strategies in the framework provided.

\section{COMMENTARY}

The environment in which we work is focused these days on the development of strategies to manage health at a systemic level. On the one hand, we are evolving new institutions and approaches to infrastructure management based on 'integrated delivery systems' and the regionalization of health services. On the other hand, we are developing strategies for the management of clinical care based on the principles of continuous quality improvement, 'evidence-based medicine' and techniques that have proved to be effective in industry in standardizing 'processes', those sequences of steps that lead to the creation of a product or a desired result. The standardization of processes appears to be fundamental to ensuring cost-effective and high-quality results in the production of any commodity, including health care. In applying this principle to the management of care on a population basis, that is, in the standardization of care and minimization of variation in practice, our fundamental tool is the 'clinical practice guideline' (CPG).

In providing us with a current CPG, Barkun and colleagues make an important contribution to our goal of standardizing the care of patients with nonvariceal upper gastrointestinal bleeding. They do this, as it should be done, by reviewing and assessing the evidence and vetting their conclusions through a panel of experts. They use a considered, formal and well-accepted approach to dealing with the available evidence. Their interpretation of the evidence undoubtedly reflects their professional experiences in dealing with such clinical problems. Support for the group's recommendations varies from complete acceptance for some (eg, development of institution-specific protocols for multidisciplinary management of gastrointestinal bleeds, including the availability of an expert endoscopist) to less enthusiastic support for others (eg, only 40\% accepted unequivocally the notion that patients awaiting endoscopy should be considered for empirical therapy with a high dose proton pump inhibitor). The issues of concern and the relevant evidence are laid out clearly for our consideration; issues that require further research are indicated.

Guidelines are most effective if they are developed by highlevel institutions that represent the recognized experts in the field, are accessible in their format and are based on credible evidence. Barkun and associates serve us well in these respects. If widely implemented, their recommendations could significantly improve outcomes for patients at the high-risk end of the clinical spectrum and improve resource use at the low-risk end. Operationalizing standards such as these, however, represents an additional challenge. Unfortunately, CPGs like these may not be as effective as we would like in achieving their intended goals (1-3).

We lack institutionalized policies for assigning authority and support for the creation, implementation and maintenance of guidelines on a systemic level; so much of this work is done on an ad hoc basis by concerned parties. Ideally, guideline development should be funded by government agencies or other 
disinterested parties. Such funding would eliminate concerns about potential bias that may arise when these undertakings are funded by industry and when industry representatives actively participate in the process of guideline development. Similarly, the dissemination and implementation of CPGs require institutional strategies that must be effective in their approach and adequately funded. Getting the message out and ensuring that it is heeded are essential for standardizing care and optimizing its quality. In addition, we must provide for the ongoing maintenance and updating of CPGs; these activities must also be appropriately funded.

It may be worthwhile to add a footnote to the guidelines Barkun et al have developed. Because the transfusion of blood involves significant risk, this intervention warrants properly informed consent before it is undertaken. Providing for fully informed consent when transfusing patients with major gastrointestinal bleeding likely represents a significant change in the manner in which some of us have dealt with these patients. This, too, is a standard of care to which we must commit.

Barkun and colleagues have provided us with useful direction regarding the management of patients with acute nonvariceal hemorrhage. We should hope that such guidelines will soon be endorsed, disseminated and implemented as part of a comprehensive and effectively managed health system, so that their full impact on health care can be realized.

\section{REFERENCES}

1. Cabana MD, Rand CS, Powe NR, et al. Why don't physicians follow clinical practice guidelines? A framework for improvement. JAMA 1999;282:1458-65.

2. Davis DA, Taylor-Vaisey A. Translating guidelines into practice: A systematic review of theoretic concepts, practical experience and research evidence in the adoption of clinical practice guidelines. CMAJ 1997;157:408-16.

3. Gundersen L. The effect of clinical practice guidelines on variations in care. Ann Intern Med 2000;133:317-8. 


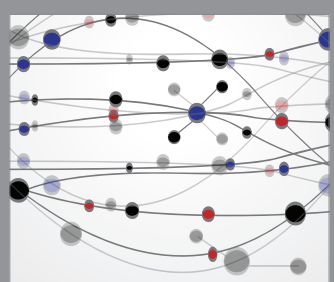

The Scientific World Journal
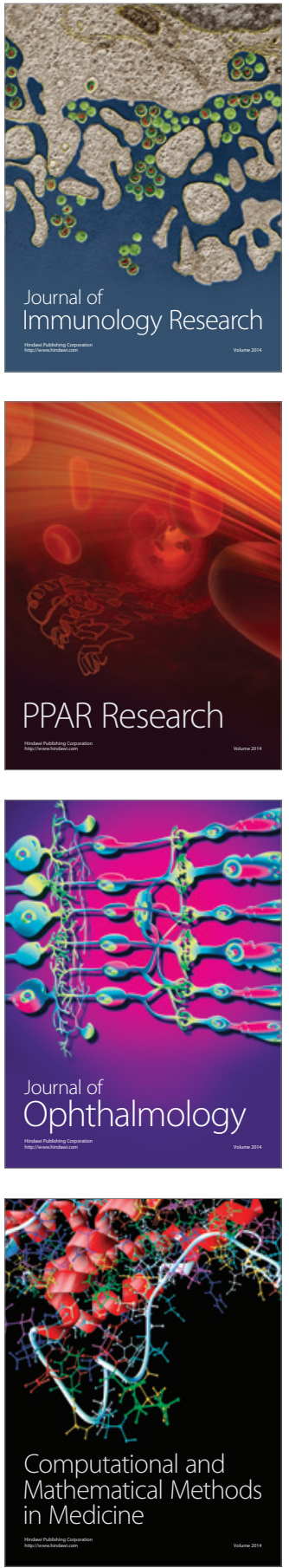

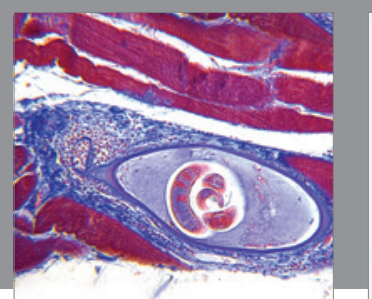

Gastroenterology Research and Practice

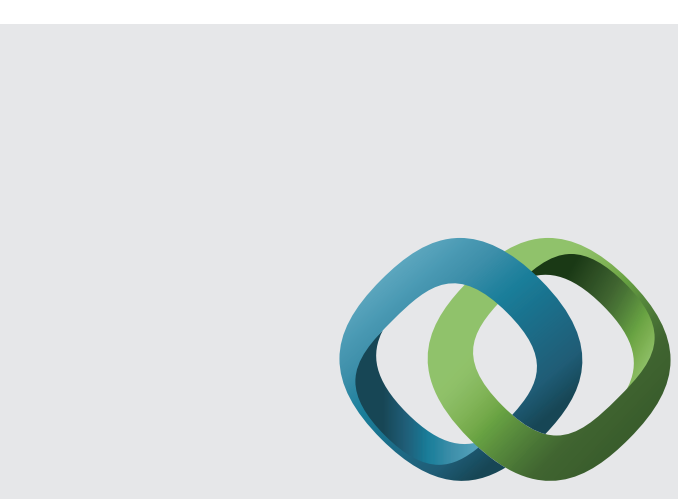

\section{Hindawi}

Submit your manuscripts at

http://www.hindawi.com
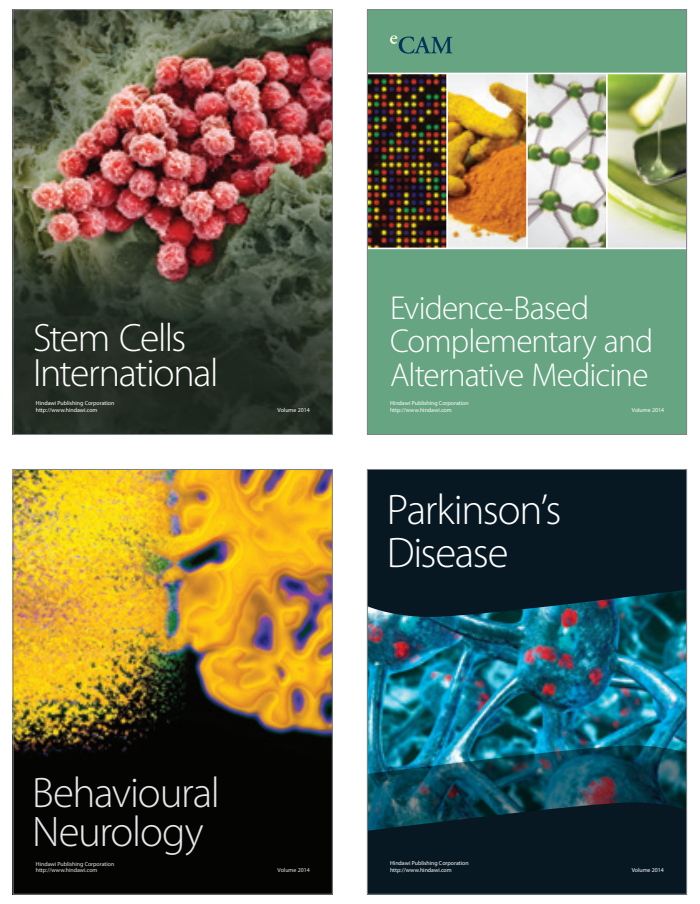
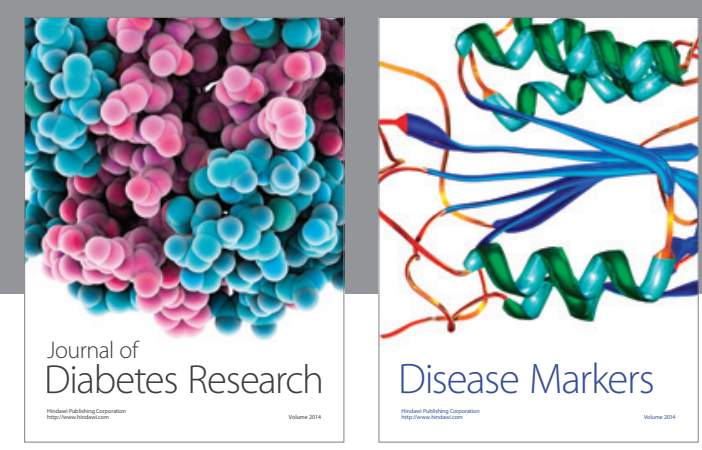

Disease Markers
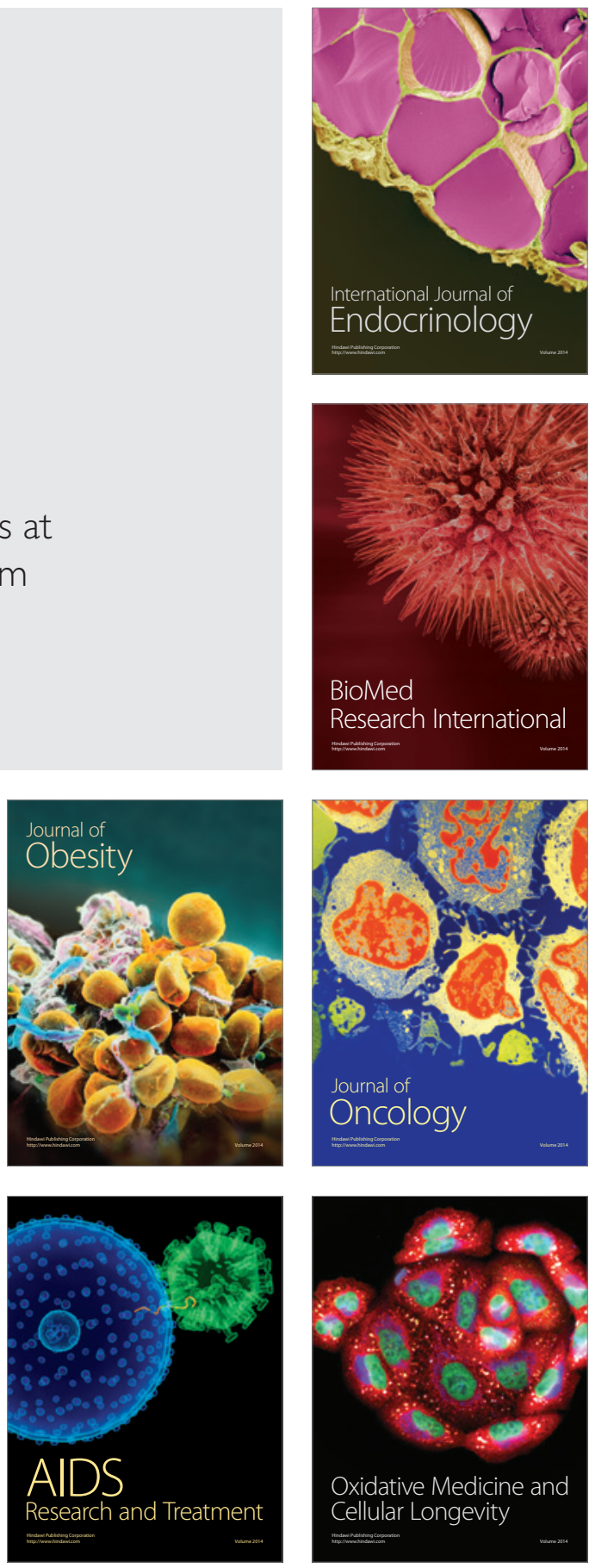